\title{
Generation of Pseudoscalar Bosons by Stimulated Raman Scattering of Light in Dielectric Media
}

\author{
V.S. Gorelik ${ }^{a}$ \\ P.N. Lebedev Physical Institute of RAS, 53 Leninskiy Ave., Moscow 119991, Russia
}

\begin{abstract}
The conditions of pseudoscalar excitations of liquids and crystals vibration states in spontaneous and stimulated Raman spectra revealing are reported. The selection rules for pseudoscalar modes of molecules and crystals observation have been obtained. The experiments on observation of spontaneous and stimulated Raman scattering on pseudoscalar modes of molecules and crystals have been fulfilled. The excitation of stimulated Raman scattering was with using of solid state laser YAG: $\mathrm{Nd}^{3+}$, generating intense (up to $1 \mathrm{TW} / \mathrm{cm} 2$ ) ultrashort $(60 \mathrm{ps})$ laser pulses with energy $10 \mathrm{~mJ}$ and frequency repetition $10 \mathrm{~Hz}$. The relationship between pseudoscalar bosons of dielectric media and axion of vacuum is analyzed.
\end{abstract}

Keywords: pseudo scalar, generation, Raman scattering, axion.

Elemental excitations of molecules and crystals are classified by irreducible presentations of corresponding symmetry groups, in accordance with the known results of group theory analyses $[1,2]$. For a number of molecular and crystalline structures in vibration spectrum there are optical modes, classified by pseudoscalar presentations with characters equal to unity for proper rotations and to minus unity for improper one (inversion, mirror place and mirror rotation). In accordance with group theory analyses for a number of cases, pseudoscalar modes are forbidden by selection rules for observation in Raman Scattering (RS) and also infrared spectra. We have analyzed group theory results of infrared, Raman and Hyper Raman selection rules for a number of liquids (water, ethanol, glycerin) and crystals (sodium nitrite, aluminum oxide, calcite, lithium niobate and some aminoacid crystals). In these media pseudoscalar modes are presented; in some substances pseudoscalar modes are "silent", i.e., are forbidden as for Raman and also for infrared spectra. In structures with $\mathrm{C}_{2 \mathrm{~h}}$ point group pseudoscalar modes are allowed for infrared but forbidden for Raman spectra. At the case sodium nitrite and some aminoacid crystals $\left(\mathrm{C}_{2 \mathrm{~V}}\right.$ point group) pseudoscalar modes are allowed for Raman but forbidden for infrared spectra. In these type structures pseudoscalar modes should have high Q-factor and high intensity in spontaneous RS. As the result the opportunity for experimental observation of stimulated Raman scattering (SRS) on pseudoscalar optical modes is opened. According to group theory predictions if outer magnetic field applying the forbidden rule is absent and pseudoscalar modes are allowed for Raman and also for infrared spectra. We have analyzed theory group predictions opportunity for Raman spectra registrations of pseudoscalar modes in water clusters, ethanol and glycerin molecules, sodium nitrite crystal, and also in aminoacid molecules and crystals. The experiments on observation of spontaneous and stimulated RS due to pseudoscalar

\footnotetext{
${ }^{\mathrm{a}}$ Corresponding author: gorelik@sci.lebedev.ru
} 
modes in sodium nitrite polycrystals, some aminoacids (L-serine, DL-tyrosine) and dried DNA have been fulfilled. As the result of experimental investigations we have registered the low frequency spontaneous Raman satellites of $\mathrm{A}_{2}$-type of $\mathrm{C}_{2} \mathrm{~V}$ point group, intensity of which were essentially higher with comparing to total symmetric ( $\mathrm{A}_{1}$-types) modes (see Fig.1 (left)).

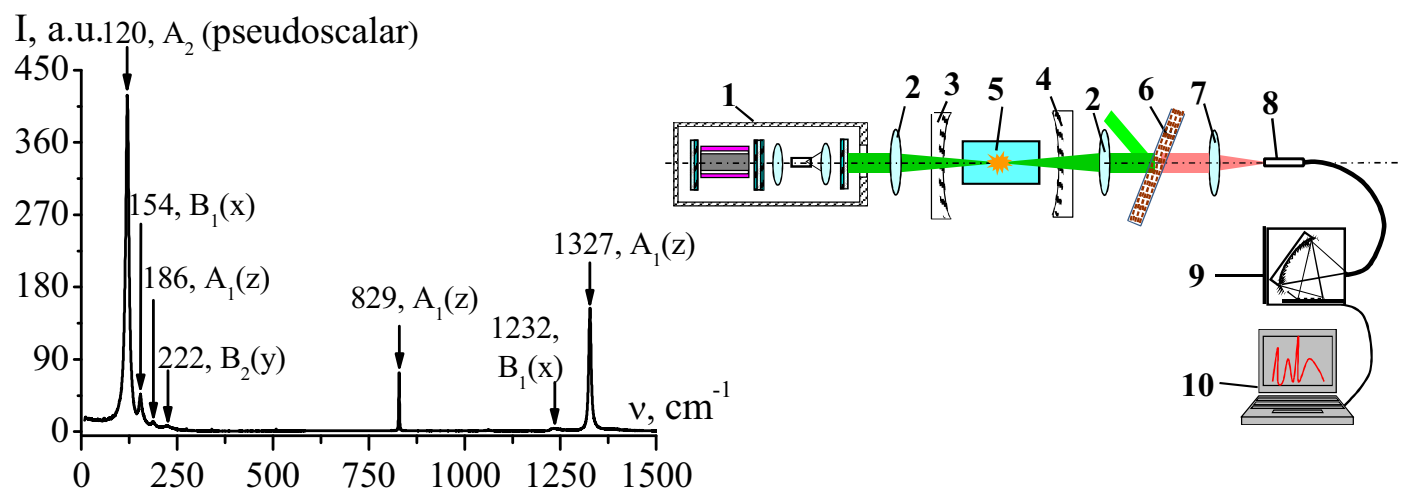

Figure 1. Left: spectrum of spontaneous RS in sodium nitrite polycrystals under continuous argon laser excitations $(\lambda=0.488 \mu)$, Maximum at $120 \mathrm{~cm}-1$ corresponds to pseudoscalar mode A2-type of $\mathrm{C} 2 \mathrm{~V}$ point group.

Right: experimental scheme for SRS observation due to pseudoscalar modes in dielectrics; 1-pulsed laser $(\lambda=0.532 \mu)$; 2-lens; 3,4- mirrors of SRS resonator; 5- focusing region of investigated dielectrics; 6- photonic crystal, reflecting of excing light emission; 7- condensor; 8-fiber; 9 - minispectrograph; 10- computer.

Experimental scheme used for pseudoscalar modes SRS observation in discussed dielectrics is presented at Fig.2. The excitation of SRS was with the help of solid state laser on YAG: $\mathrm{Nd}^{3+}$, working with the conversion of the basic emission at $\lambda=1.064 \mu$ into green radiation at $\lambda=0.532 \mu$. The generation of intense (up to $1 \mathrm{TW} / \mathrm{cm}^{2}$ ) ultrashort (60ps) laser pulses with frequency repetition $10 \mathrm{~Hz}$ and energy value of every pulse equel to $10 \mathrm{~mJ}$ was realized. In SRS spectra of investigated samples the intense Stokes and anti Stokes satellites at low frequency region have been observed. Such intense satellites correspond to coherent pseudoscalar modes (bosons) in dielectric media. The relationship between pseudoscalar waves being generated under SRS in dielectric media with vacuum axion waves, the prediction of which is widely discussed recently in literature [1-3], has been analyzed. Principal experimental schemes for photon-axion conversion processes under the presence of outer magnetic field are presented. The opportunity of "Light Shining Trough Wall" phenomenon (Primakoff effect [4]) in dielectric media under SRS has been discussed

\section{References}

1. L.D. Duffy, P. Sikivie, D. B. Tanner,S. J. Asztalos, C. Hagmann, D. Kinion, L. J Rosenberg, K. van Bibber, D. B. Yu, R. F. Bradley, Physical Review D. 74. 012006 (2006)

2. P. Sikivie, D.B. Turner, and Karl van Bibber, Phys. Rev. Lett. 98.172002 (2007)

3. D.D.Stancil, Physical Review D. 76. 111701(R) (2007)

4. J. E. Kim, Rev. Mod. Phys. 82, No. 1. P. 557-601 (2010) 